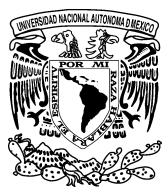

\title{
Contabilidad de gestión para controlar o coordinar en entornos turbulentos: su impacto en el desempeño organizacional
}

\author{
Management accounting for controlling or coordination in turbulent \\ environments: Their impact on organizational performance
}

\author{
Marcela Porporato \\ School of Administrative Studies, York University, Canadá \\ Recibido el 6 de mayo de 2014; aceptado el 8 de febrero de 2015 \\ Disponible en Internet el 2 de julio de 2015
}

\begin{abstract}
Resumen
Este trabajo analiza si la información generada por los sistemas de contabilidad de gestión (SCG) impacta de modo diferente sobre el desempeño de organizaciones en entornos turbulentos según se use para controlar o coordinar (conceptos de la teoría de costos de transacción). Los datos provienen de una encuesta con 42 respuestas de empresas localizadas en la provincia de Córdoba, Argentina. Los resultados destacan el efecto positivo sobre el desempeño organizacional cuando la contabilidad de gestión se emplea principalmente para coordinar en entornos turbulentos hasta un cierto límite. El propósito de uso se modela mejor como variable independiente y no como variable mediadora o moderadora. Estudios de casos indicaron que los SCG median los factores externos y el desempeño, pero aquí se refuta la idea aplicada a entornos turbulentos donde las acciones de los directivos no influyen sobre factores externos (mercado y tecnología). Se sugiere, para empresas medianas en economías regionales de Latinoamérica, que si los SCG se usan para coordinar se tendrá un efecto positivo en el desempeño organizacional, combinado con el uso para controlar limitado a medición de costos, sistemas de compensación e incentivos.

Derechos Reservados ( 2015 Universidad Nacional Autónoma de México, Facultad de Contaduría y Administración. Este es un artículo de acceso abierto distribuido bajo los términos de la Licencia Creative Commons CC BY-NC-ND 4.0.
\end{abstract}

Palabras clave: Contabilidad de gestión; Desempeño organizacional; Encuesta; Propósito de uso; Argentina

Correo electrónico: porpomar@yorku.ca

La revisión por pares es responsabilidad de la Universidad Nacional Autónoma de México. 


\begin{abstract}
This study explores management accounting systems' (MAS) effect on organizational performance in turbulent environments. The information generated by MAS has a different impact on organizational performance depending on its main purpose of use (control or coordination) according to transaction costs theory. Data from a survey with 42 complete answers from medium sized organizations operating in the province of Cordoba (Argentina) show that MAS positively impact organizational performance in turbulent environments if used to coordinate up to a certain level. Their purpose of use is better modeled as another independent variable but not as a mediator between external factors and organizational performance. Previous case studies suggested that MAS's purpose of use was mediating between external factors and organizational performance, but this study shows that in turbulent environments the idea is not valid as managers' decisions do not affect uncontrollable external factors (market and technology). Performance in medium sized organizations operating in Latin America improves if MAS are used to coordinate, while limiting their use for control purposes to certain and definite tasks such as cost measurement, compensation and incentive mechanisms.
\end{abstract}

All Rights Reserved () 2015 Universidad Nacional Autónoma de México, Facultad de Contaduría y Administración. This is an open access item distributed under the Creative Commons CC License BY-NC-ND 4.0 .

Keywords: Management accounting; Organizational performance; Survey; Purpose of use; Argentina

\title{
Introducción
}

El modo en que se usa la información generada por el sistema contable impacta de modo diferente en el desempeño organizacional, especialmente en ámbitos turbulentos como Latinoamérica. La teoría de costos de transacción sostiene que los sistemas de la contabilidad de gestión (SCG) cumplen 2 propósitos, uno es coordinar entre agentes y principales, y el otro es ser usados por el principal para controlar las acciones del agente. Este estudio evalúa el efecto en el desempeño organizacional según el uso que se haga de los SCG, control versus coordinación, mediante una encuesta en un entorno turbulento. La definición de entorno turbulento se toma prestada de la literatura sobre administración o gestión estratégica (strategic management) y se ha definido como un entorno cambiante, volátil e impredecible (Camillus y Datta, 1991; Grant, 2003).

La literatura sobre contabilidad y administración ha documentado que la contabilidad de gestión ayuda a reducir la incertidumbre, pero aún se discute si mejora o no el desempeño de las organizaciones (Wang, Wee y Koh, 1998). La teoría existente permite concretar que la información reduce la incertidumbre entendida como «la diferencia entre la cantidad de información requerida para desarrollar la tarea y la cantidad de información disponible en la organización» (Galbraith, 1973, p.5); pero estos sistemas pueden ser rígidos, no permitiendo a las organizaciones reaccionar rápidamente (Davila, 2000). Se especula que el exceso de rigidez solo sucede en entornos estables, pero está poco documentado que ese efecto secundario nocivo exista en entornos turbulentos (Kattan, Pike y Tayles, 2007) y particularmente en Latinoamérica (Porporato y Garcia, 2011).

Este trabajo está organizado en 5 secciones que incluyen esta introducción y conclusión. La primera sección ofrece una introducción al tema donde es expuesta la motivación del estudio. La segunda sección es una revisión de la literatura en general que ubica este estudio dentro de la literatura de contabilidad; se ofrece una descripción del modelo general explicitando las hipótesis a contrastar. La tercera sección explica la metodología empleada en la porción empírica 
del estudio; se pone especial énfasis en explicar las decisiones del diseño de la investigación y la medición de las variables. La cuarta sección presenta los resultados obtenidos al aplicar métodos estadísticos a los datos recogidos en la encuesta. El trabajo acaba con la discusión de los resultados y una conclusión general.

\section{Revisión de literatura e hipótesis}

Las bases teóricas de este estudio provienen de la teoría de costos de transacción y de la teoría de la contingencia. La teoría de costos de transacción explica por qué la información ayuda a reducir la incertidumbre y la teoría de la contingencia permite armar un modelo que puede ser evaluado empíricamente. La teoría de costos de transacción nos presenta 2 formas de entender el rol de los SCG; por un lado hablamos de instrumentos de control que reducen la divergencia de los intereses entre los miembros de la organización (control), y por otro, nos referimos a la infraestructura de información que los miembros de la organización utilizan para resolver la incertidumbre que enfrentan en sus decisiones (coordinación).

\section{Teoría de costos de transacción y propósito de uso de los sistemas de la contabilidad de gestión}

Los reportes generados por los SCG son usados tanto para evaluar el pasado como para tomar decisiones que afectarán el futuro. El primer propósito de los SCG es salvaguardar los activos específicos invertidos en la firma (Williamson, 1985) y el segundo es coordinar tareas complejas (Thompson, 1967). Si al concepto anterior se lo considera a la luz de la definición de incertidumbre de Galbraith (1973), la contabilidad de gestión es un sistema de información que ayuda a tomar decisiones pero también es un instrumento de control posterior. Esta tensión entre información para la toma de decisiones (uso orientado al futuro) y para el control (uso orientado al pasado) surge porque los datos y mecanismos que ayudan a establecer controles no son necesariamente los mejores para la toma de decisiones (Davila, 2000). La díada conflictiva que contrapone el uso de la información para la toma de decisiones y para el control es un concepto prevalente en la literatura de contabilidad (Demski et al., 2002).

Revisando la literatura contable y de administración no queda claro si los SCG mejoran o empeoran el desempeño de las empresas. El mayor argumento esgrimido es que ayudan a reducir la incertidumbre sobre la información necesaria para tomar una acertada decisión (Davila, 2000), pero crean un chaleco de fuerza que demora la reacción de las organizaciones lo que tendría consecuencias negativas en entornos turbulentos (Kattan et al., 2007). Los SCG no se manifiestan claramente en las organizaciones, por ello se estudian a 2 niveles que son contrastables empíricamente (Davila, 2000). Primero los mecanismos tales como los presupuestos o medición del desempeño e incentivos, y segundo, el tipo de información que capturan y transmiten, tales como los indicadores financieros o de clientes.

\section{Teoría de la contingencia: modelo de relaciones}

La teoría de la contingencia permite relacionar factores externos que condicionan el desempeño organizacional. En la literatura se ha indicado que el desempeño organizacional está determinado por el mercado y la naturaleza del trabajo realizado. Esta teoría sugiere que las particularidades del entorno y tareas en la organización generan el desarrollo de estrategias para coordinar y controlar las actividades internas. Más específicamente, según estudios previos, cuando el uso 
balanceado de los SCG para coordinar y controlar coinciden con el entorno y las tareas a realizar, el desempeño de la organización mejora (Drazin y van de Ven, 1985).

La literatura en contabilidad de gestión registra una gran cantidad de estudios que han empleado la teoría de la contingencia para desarrollar y probar empíricamente diversos modelos de relaciones (Chenhall, 2007). Varios factores contingentes internos y externos han sido utilizados para explicar la combinación de prácticas y procesos de coordinación y control que emplean las organizaciones (Thompson, 1967; Galbraith, 1973). Por ello se relacionan el perfil y características de la firma (influidos por factores contingentes considerados como fuentes de incertidumbre) con las decisiones en el diseño de SCG (reflejadas en el propósito de uso de los tipos de información y componentes). El primer factor analizado muestra que el entorno afecta el diseño de los SCG (Chenhall y Morris, 1986; Govindarajan, 1988; Simons, 1990) y afecta selectivamente el uso de la información (Davila, 2000). Pocas aplicaciones hay en entornos turbulentos, pero Kattan et al. (2007) indican que cuanto mayor sea la experiencia menor será la complejidad percibida, por lo que la incertidumbre se reduce debido a un uso eficiente de la información disponible, mientras que Porporato y Garcia (2011) no encuentran relación entre la intensidad de uso de la información y factores externos percibidos como no controlables por la organización.

El segundo factor que afecta el diseño de los SCG es la tecnología que se ha caracterizado según tamaño, número de excepciones en el proceso de producción, y tipo de relaciones entre procesos: conjuntos, secuenciales y recíprocas (Thompson, 1967). La relación probada en la literatura de contabilidad muestra que la predictibilidad de la tarea afecta el diseño de los SCG (Hayes, 1977; Waterhouse y Tiessen, 1978; Chenhall y Morris, 1986) e impacta selectivamente el uso de la información (Davila, 2000). Los estudios que han cubierto este tema en entornos turbulentos son limitados, pero los casos estudiados por Kattan et al. (2007) y Porporato y Garcia (2011) indican que cuanto mayor sea la experiencia menor será la complejidad percibida y la incertidumbre debido a un uso eficiente de la información disponible.

\section{Hipótesis}

A pesar de la complejidad y particularidad de cada factor considerado, todas las relaciones pueden colapsarse, sosteniendo que mientras mayor sea la experiencia con los factores que se perciben como controlables por la gerencia, los SCG serán usados más bien orientados a coordinar que a controlar. Las firmas que operan en un entorno turbulento conocen las necesidades y requerimientos de sus «stakeholders»; frecuentemente las necesidades y requerimientos cambian y la incertidumbre sobre los requisitos e información para evaluarlos aumenta, transformándose en poco controlable por las decisiones gerenciales. La experiencia les permite a los gerentes saber el tipo de información a utilizar y cómo emplearla para mejorar el desempeño, por ello se reduce la incertidumbre cuando la información se usa más bien para coordinar. De modo similar, el producto y la tecnología del proceso pueden oscilar entre ser muy conocidos hasta ser totalmente desconocidos, implicando en el primer caso una fuente de incertidumbre controlable si se dispone de información para coordinar (McGrath, 1995). Estos razonamientos permiten elaborar las siguientes hipótesis escritas en el modo alternativo y unilateral:

H1a. Organizaciones con entornos altamente turbulentos usarán los SCG principalmente para controlar.

H1b. Organizaciones con tareas altamente turbulentas usarán los SCG principalmente para controlar. 
Según como se entienda el uso de los SCG se puede explicar un efecto positivo o negativo sobre el desempeño organizacional. Si la información se usa para controlar el desempeño a posteriori (proteger activos invertidos), se observa que las personas actúan defensivamente frente a la información tratando de justificar sus decisiones pasadas y evitan compartir información actual o datos muy detallados por temor a que en el futuro sea empleada para juzgar y sancionar sus decisiones (Vosselman y van der Meer-Kooistra, 2009). Por el contrario, si la información se usa para coordinar las decisiones actuales dentro de las unidades de negocio, se genera un entorno relajado en donde las personas comparten la información y datos tanto financieros como no financieros porque el objetivo es mejorar el desempeño organizacional y no protegerse de futuros juicios de valor sobre las decisiones tomadas (Vosselman y van der Meer-Kooistra, 2009). La relación entre el uso de los SCG y el desempeño es positiva si la empresa cuenta con un proceso de información estructurado y compartido que se utilice intensivamente para reducir la incertidumbre y facilitar la coordinación entre diversos actores. Por otro lado, procesos e informes muy estructurados probablemente tengan un efecto negativo en la habilidad de la gerencia para responder a circunstancias cambiantes. Sin embargo, la existencia de conductas oportunistas puede ser prevenida utilizando los SCG con el propósito de controlar. La hipótesis 2 escrita en el modo alternativo y unilateral es:

H2. En organizaciones con tareas turbulentas que operan en entornos turbulentos, los SCG que afectan positivamente el desempeño de las empresas se utilizan principalmente para controlar.

\section{Decisiones que afectan el diseño de investigación}

Una serie de decisiones en el diseño de la investigación permiten ofrecer resultados consistentes. La primera decisión es el tipo de organización escogido, ya que el propósito de este trabajo es encontrar una explicación para el impacto de la contabilidad de gestión en el desempeño de organizaciones que operan en entornos turbulentos. Un fenómeno atractivo para estudiar, debido a los escasos estudios preexistentes, es la realidad de las empresas localizadas en economías regionales en países emergentes, siendo las industrias manufactureras medianas de la provincia de Córdoba (Argentina) el caso escogido.

Las contribuciones de este estudio pueden ser extendidas a otras situaciones si se observan algunas restricciones. Primero, el estudio empírico se concentra en industrias de Córdoba, por lo tanto las conclusiones pertenecen a este tipo de organizaciones en particular. La decisión de limitar los casos a un subgrupo particular de industrias se basa en el hecho de que empresas de servicios o industrias muy diversas pueden diferir apreciablemente en la complejidad de la tecnología, estructura, procesos y procedimientos de administración (Wang et al., 1998). Segundo, los datos en cada empresa se obtuvieron mediante encuestas que son informes verbales susceptibles a los efectos nocivos de recuerdos limitados o selectivos y articulación pobre o inexacta.

\section{Unidad de análisis}

Una decisión esencial es la definición de la unidad de análisis. Anthony (1965) identificó 3 niveles de SCG que se consideran pertinentes para el propósito de este estudio: estratégico, operativo y táctico. Idealmente la unidad de análisis es la persona que ocupa una posición en la organización que le obligue a trabajar con los 3 niveles hasta cierto punto. Basándose en estudios previos, el gerente general (socio-gerente) está directamente implicado con los SCG 
en los 3 niveles, por consiguiente esta persona será la unidad de análisis de este estudio y nos enfocaremos en los SCG que él/ella utiliza de un modo regular.

\section{Pruebas de certeza y validez.}

La medición de ítems incluidos en los estudios empíricos y su transformación en variables se debe someter a pruebas de certeza y validez. La medición se enfoca en la relación entre los indicadores de los estudios empíricos (las respuestas observables) y los conceptos ocultos fundamentales (conceptos teóricos). Si esta relación es significativa, el análisis de indicadores empíricos puede llevar a inferencias útiles acerca de las relaciones entre los conceptos teóricos fundamentales. Las fuentes comunes de error fueron controladas utilizando preguntas ya empleadas en otros estudios; encuestas similares, pero en inglés, se reportan en el estudio de Davila (2000) respecto a SCG y de Ariño (2003) respecto al desempeño organizacional en un entorno turbulento como son las alianzas estratégicas. Para asegurarse de que la traducción de ítems de los cuestionarios era adecuada, se requirió a una tercera persona que hiciera una traducción inversa (del español al inglés) de la encuesta que iba a ser usada para este estudio. Los instrumentos de Davila (2000) y Ariño (2003) en inglés fueron comparados con la traducción inversa y no se encontraron diferencias significativas. Para evitar errores de percepción, el cuestionario revisado luego de la traducción inversa fue pretesteado con 3 sujetos, todos ellos socios o gerentes de empresas industriales localizadas en Argentina. En este trabajo la medida de consistencia interna considerada para la mayoría de las medidas es el alfa de Cronbach (Cronbach, 1951), porque es más conservadora ya que representa el límite inferior de la certeza de una escala no proporcional de $\mathrm{N}$ ítems, esto es, $\mathrm{p} \geq \alpha$ (Carmines y Zeller, 1979). Se han controlado errores de no respuesta y de respuesta tardía.

\section{Definición de variables usadas en el estudio empírico}

\section{Variable dependiente: desempeño organizacional}

El desempeño es un concepto complejo y multidimensional; estudios previos sugieren que se deben incluir múltiples medidas del desempeño para capturar las diferentes dimensiones. Existe evidencia de que las medidas objetivas del desempeño no capturan completamente las complejas metas que se fijan, por eso se han introducido medidas de percepción tales como el logro de los objetivos de los socios. Siguiendo el diseño de Davila (2000) y Ariño (2003) el desempeño puede ser valorado como alto o bajo basado en la opinión de los gerentes mediante 13 ítems en el cuestionario. El inconveniente de utilizar medidas basadas en opiniones sobre la empresa que se gerencia es que son afectadas por la percepción del sujeto, pero tiene la ventaja de capturar la mayor parte de las dimensiones pertinentes y las expectativas. En 2 organizaciones el desempeño informado por los gerentes fue contrastado con el desempeño financiero informado por la firma a terceros (datos públicamente disponibles). En ambos casos se encontró que había una concordancia alta entre ambos grupos de indicadores. Los 13 ítems que componen esta variable están altamente relacionados en un solo factor (67\% de la variación) y son colapsados en una sola medida calculada como un promedio para mantener la relevancia y comparabilidad de la escala; esta medida tiene alto coeficiente de certeza (alfa de Cronbach de 0.8818). Los detalles del cuestionario y conformación de factores están disponibles en el Apéndice, parte 1 . 


\section{Sistemas componentes de la contabilidad de gestión}

Cinco son los componentes de los SCG identificados en industrias cordobesas. Estos 5 tipos de componentes ya fueron empleados en estudios anteriores (Davila, 2000; Porporato y Garcia, 2011). La siguiente es una descripción de cada uno:

1) Mecanismos de gobierno. Mecanismos institucionales por los que las relaciones organizacionales son iniciadas, negociadas, diseñadas, coordinadas, controladas, adaptadas, y terminadas. La mayor parte de los estudios están dentro de la literatura de administración y se concentran en el modo en el que los mecanismos de gobierno corporativo se emplean para controlar. La definición de esta variable sigue los lineamientos de Groot y Merchant (2000) y Dekker (2004).

2) Asignación de costos. Procedimientos por medio de los cuales los costos del producto se construyen. La asignación de costos y la determinación de precios se relacionan generalmente bajo los conceptos de centros de responsabilidad y precios de transferencia (Kaplan, 1998; Anthony y Govindarajan, 2007; Merchant y van der Stede, 2011), alternativamente se relacionan con la evaluación del desempeño (Demski, 2008). En este trabajo se adopta el primer enfoque, con un uso para coordinar. Las dimensiones escogidas de esta variable se basan en Kaplan (1998) y Demski (2008).

3) Planeamiento y presupuestación. Las múltiples funciones del presupuesto (autorizar, pronosticar, planificar, comunicar, coordinar, motivar, evaluar y controlar) provocan consecuencias disfuncionales como la desconfianza, resistencia, y conflicto interno (Shields, 1997). Los presupuestos son un instrumento básico para promover la coordinación, ya que los controles posteriores basados en ellos están incluidos en el subsistema de medición del desempeño. Brownell (1985) proporciona las dimensiones pertinentes de esta variable.

4) Medición del desempeño y compensación. El desempeño se evalúa cuando se hace un plan con metas y en una fecha posterior se mide el logro de esa meta (Demski, 2008), por ello la medición del desempeño se usa para controlar. Kren y Liao (1988) presentan las dimensiones que se utilizan en este estudio.

5) Fijación de precios externos e internos (transferencia). Los precios de transferencia se fijan internamente para registrar la transferencia de bienes o servicios entre las divisiones de la misma firma e idealmente deben ser semejantes a los precios que se cargarían si el producto fuese vendido a clientes externos o comprado de vendedores externos (Anthony y Govindarajan, 2007). Las dimensiones de esta variable se desarrollan siguiendo las categorías teóricas de Ronen y McKinney (1970), Kaplan (1998) y Simons (2000).

Los estudios previos muestran una discrepancia respecto a los mecanismos de la contabilidad de gestión considerados en cada tipo de literatura (la literatura de administración considera principalmente los mecanismos de gobierno, y la de contabilidad de gestión considera los otros 4). Quizás esa discrepancia indique una perspectiva diferente; por ejemplo, la literatura de administración está enfocada en mecanismos de control para la alta gerencia, por lo que los trabajos en esta literatura sostienen que el control afecta negativamente el desempeño (Boateng y Glaister, 2002). Mientras que la literatura de contabilidad de gestión contempla mecanismos de control de gestión para niveles medios y altos de gerencia lo que introduce el uso con propósito de coordinar, lo que ayuda a reducir la incertidumbre y mejorar el desempeño (Davila, 2000; Groot y Merchant, 2000; Dekker, 2004). 
Tipos de información de la contabilidad de gestión

Este trabajo se enfoca en 5 tipos de información identificados como frecuentemente utilizados en industrias cordobesas. Estos 5 tipos de información ya fueron empleados en estudios anteriores (Davila, 2000). La siguiente es una descripción de los tipos de información considerados:

1) Regulaciones locales: son indicadores que reflejan los asuntos que cambian cuando se modifica el poder político del país o aquellos que están permanentemente bajo vigilancia, tales como leyes, normativa impositiva, seguridad laboral, medio ambiente, etc.

2) Medidas relacionadas con el tiempo: todo aquello que permite seguir los tiempos de cada producto o proceso. Si hablamos de componentes ya en producción, las medidas se asocian con el establecimiento de la línea de producción y la planificación logística, reflejando si la línea de producción trabaja a una velocidad adecuada. Si hablamos de componentes en la fase de diseño, las medidas apuntan a seguir la culminación con éxito de cada fase del desarrollo según las fechas acordadas.

3) Medidas financieras: son las medidas más típicas incluidas en el sistema de información contable y se utilizan para evaluar cuán bien o mal se están ejecutando las operaciones; estas incluyen presupuestos, costos, informes y análisis de margen y capacidad de ganancia.

4) Medidas de cliente y mercado: incluyen información sobre los requerimientos de los clientes (las cantidades a remitir periódicamente se basan en el plan acordado) o información del mercado acerca de las ofertas para cotizar nuevos productos.

5) Medidas de desempeño tecnológico de los productos o procesos (conformidad general de calidad) y las medidas de eficiencia. Entre estas medidas se incluyen las especificaciones (planos) de cada producto y la identificación de los proveedores y de sus componentes para proporcionar la certeza de la calidad y funcionalidad requeridas.

\section{El propósito de uso de los sistemas de la contabilidad de gestión}

Tal como se ha discutido, este estudio diferencia 2 propósitos en el uso de los SCG: control y coordinación. Siguiendo a Davila (2000) se mide utilizando una escala de 5 puntos tipo Likert donde en un extremo se menciona «solo para controlar (1)» y en el otro extremo se menciona «solo coordinación (5)». Las medidas se agrupan en 2 variables: propósito de uso de los componentes del SCG (3 ítems por cada componente presentado en la sección Sistemas componentes de la contabilidad de gestión) y propósito de uso de los distintos tipos de información (un ítem por cada tipo de información presentada en la sección Tipos de información de la contabilidad de gestión).

La figura 1 muestra el análisis factorial de los 20 ítems del cuestionario (propósito de uso según el tipo de información: ítems 1 a 5; y según los componentes del SCG: ítems 6 al 20). El primer factor explica el $49 \%$ de la variación en las industrias cordobesas. El gráfico muestra una clara diferencia entre control y coordinación, pero pareciera que la coordinación se refiere a 2 horizontes de tiempo diferentes: estratégica de largo plazo y operativa de más corto plazo; también es de destacar que el ítem 3 (resultados financieros) es el único que puede ser identificado con los 3 grupos. Para operacionalizar esta variable, los ítems han sido colapsados en una sola variable, que se calcula como un promedio simple, lo que es una adecuada representación del concepto que se quiere medir (alfa de Cronbach de 0.7661). Los detalles del cuestionario y conformación de factores están disponibles en el Apéndice, parte 2.

\section{Variables independientes: Factores externos}

Se consideran 2 variables contingentes: volatilidad del entorno y complejidad de la tarea. La volatilidad del entorno incluye todo aquello que está fuera de las fronteras de la organización sea o 


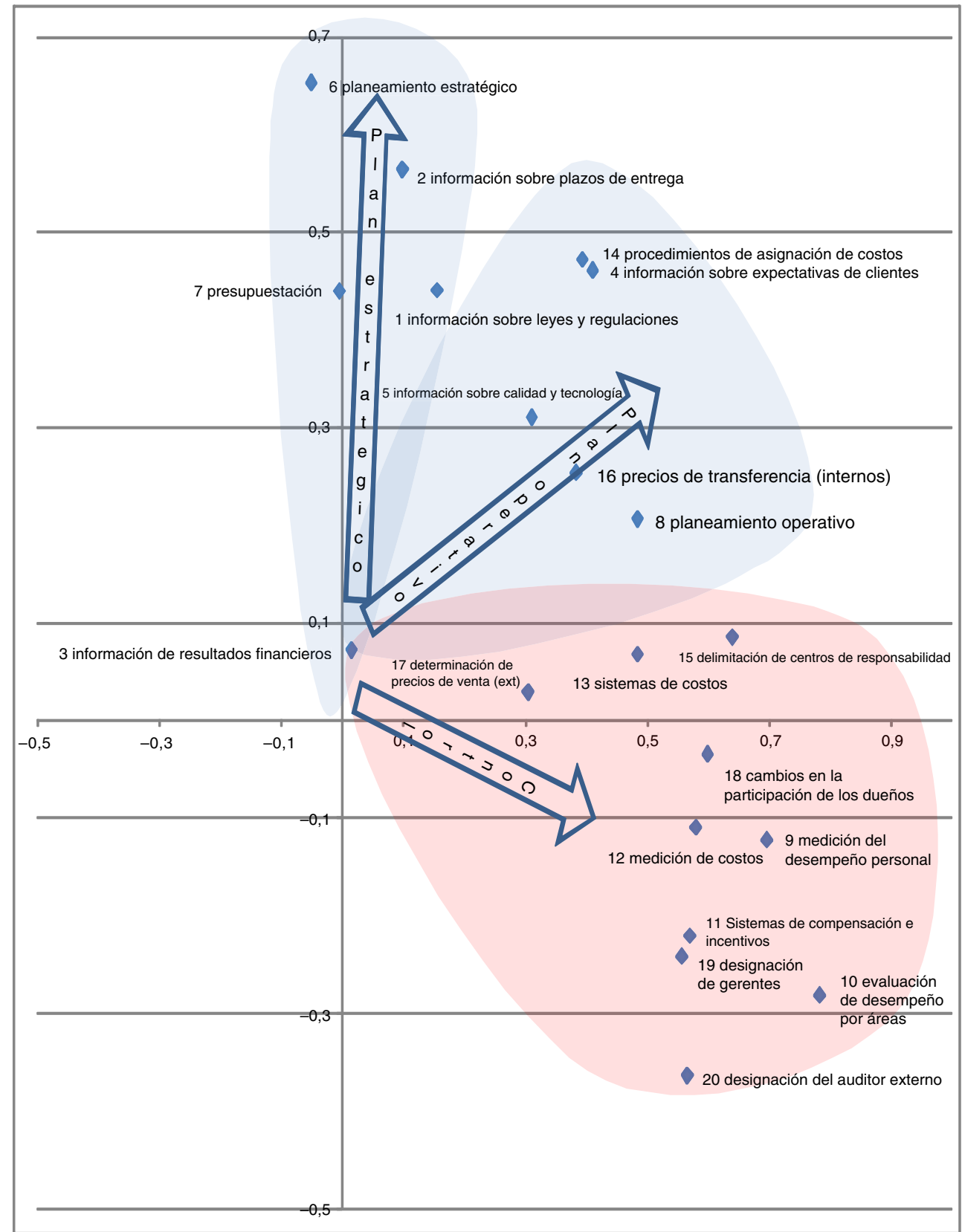

Figura 1. Propósito de uso de los SCG-Análisis factorial.

no controlable por los gerentes tales como: cambios del entorno (Hofstede, 1981), inestabilidad y complejidad del mercado (Khandwalla, 1972), y precio y producto (Gordon y Narayanan, 1984). En este trabajo la volatilidad del entorno mide la percepción de la experiencia que se tiene con el mercado local, con la industria y con los competidores en general. Para lograr un modelo válido, 
este trabajo se enfoca en la volatilidad del entorno que describe la importancia de la experiencia con el mercado local en primer lugar y con la industria suplementariamente (Von Hippel, 1998). La complejidad de la tarea se caracteriza como la complejidad de la tecnología que se mide de acuerdo a la percepción de experiencia con la misma; por lo tanto se la define en términos de experiencia con el proceso de producción y la tecnología del producto. Los ítems del cuestionario son colapsados en una sola medida para cada factor externo (volatilidad del entorno: 9 ítems, alfa de Cronbach 0.7196; y volatilidad de la tarea: 12 ítems, alfa de Cronbach 0.8398). Los detalles del cuestionario y conformación de factores están disponibles en el Apéndice, parte 3.

\section{Variables de control}

El objetivo de incluir 3 variables de control es ver si los modelos siguen siendo válidos cuando se incorporan conceptos que la literatura de administración sostiene que son factores críticos para el éxito y supervivencia de las organizaciones. La primera variable de control es el tamaño o estructura organizacional y en este estudio se incluyen 2 indicadores: superficie de la planta y año de inicio de actividades. La segunda variable de control es la importancia de los objetivos estratégicos para las industrias cordobesas ( 5 ítems, con alfa de Cronbach de 0.7912). Para la tercera variable de control este estudio considera el compromiso de los socios como un subrogante de la variable confianza (trust), entendida como confiar lo suficiente en un socio o una idea como para invertir valiosos recursos a pesar del riesgo inherente. La confianza es medida como la inversión hecha en cada organización en términos de personal, instalaciones, tiempo y dinero (4 ítems, con alfa de Cronbach de 0.7145). Los detalles del cuestionario y conformación de factores están disponibles en el Apéndice, parte 4.

\section{Selección de la muestra}

Para la encuesta se estima que existen 3,121 establecimientos industriales en la provincia de Córdoba $^{1}$, sin embargo muchos de ellos son calificados como pequeños talleres por lo que no se consideran como parte de la población. La encuesta logró una tasa de respuesta del 12\% (45 respuestas completas de 388 cuestionarios enviados) lo que se logró siguiendo varios procedimientos (Dillman, 2009).

\section{Resultados}

La medición de los ítems incluidos en la encuesta y su transformación en variables se ha sometido a pruebas de certeza y validez. Para comprobar la no existencia de errores por falta de respuestas y autoselección de los participantes, la muestra se partió en mitades y no se encontraron diferencias significativas entre ambas tanto en la estadística descriptiva como en las regresiones en las que el desempeño es la variable dependiente. Adicionalmente, el uso del análisis factorial contribuye a mejorar la certeza y validez (Carmines y Zeller, 1979).

El gerente general de la organización es la unidad de análisis de este estudio y nos enfocamos en los SCG que ellos utilizan normalmente. Los gerentes reciben información con más frecuencia que la que ellos generan para otros actores y las diferencias son estadísticamente significativas

\footnotetext{
${ }^{1}$ Secretaría de Industria y Comercio de la Provincia de Córdoba, consultada en mayo de 2006.
} 
Tabla 1

Perfil de las empresas participantes $(n=42)$

\begin{tabular}{llll}
\hline Características & Media & Mínimo & Máximo \\
\hline Número de empleados & 113 & 43 & 535 \\
Porcentaje de empleados de producción & $74.4 \%$ & $30 \%$ & $97 \%$ \\
Niveles jerárquicos & 3.64 & 2 & 6 \\
Líneas de producción & 2.60 & 1 & 6 \\
Número de diferentes productos & 3.88 & 2 & 7 \\
Cantidad de plantas & 1.24 & 1 & 7 \\
Ventas anuales (millones de pesos) & Entre 1 y 5 & Menos de 0.5 & Más de 100 \\
\hline
\end{tabular}

(t-test) por lo que se justifica claramente la selección del gerente general como la unidad de análisis.

\section{Estadísticas descriptivas}

La tabla 1 resume el perfil de las empresas participantes mientras que la tabla 2 presenta las estadísticas descriptivas de todas las variables usadas en este estudio. Si bien se recibieron 45 respuestas completas, 3 de ellas fueron eliminadas del análisis por 2 razones. Una respuesta fue eliminada dado su tamaño y complejidad que la hacen no comparable con el resto de las respuestas recibidas $\left(200,000 \mathrm{~m}^{2}\right.$ tiene la planta, y cuenta con más de 1,100 empleados); otras 2 respuestas fueron eliminadas al indicar que estaban por cesar operaciones debido a pobres resultados obtenidos (próximas a solicitar su quiebra). La matriz de correlación entre variables independientes muestra una sola relación significativa al 10\%. En las industrias cordobesas la inversión está relacionada con la importancia dada a los objetivos estratégicos, reflejando una relación lógica entre los recursos dedicados a un emprendimiento que se percibe como importante.

\section{Resultados: Factores externos y el uso de los sistemas de la contabilidad de gestión}

Para probar las hipótesis que relacionan los SCG con factores externos, este trabajo usa la siguiente ecuación de regresión:

Propósito de uso de los $\mathrm{SCG}=f$ (factores externos)

La tabla 3 muestra los resultados de 2 regresiones múltiples del tipo OLS que intentan capturar el efecto de los factores externos en el uso de los SCG. La hipótesis H1a predecía una relación

Tabla 2

Estadísticas descriptivas de las variables

\begin{tabular}{llllll}
\hline Variables & $\mathrm{N}$ & Media & DE & Rango actual & Rango teórico \\
\hline Desempeño & 42 & 3.2784 & 0.5244 & $2.30-4.92$ & $1-5$ \\
Propósito de uso de los SCG & 42 & 2.4302 & 0.5152 & $1.13-3.40$ & $1-5$ \\
Volatilidad del entorno local & 42 & 3.2857 & 0.5036 & $1.55-4.55$ & $1-5$ \\
Volatilidad de la tarea & 42 & 2.9266 & 0.6030 & $1.16-4.50$ & $1-5$ \\
Inversión & 42 & 3.3691 & 0.6029 & $1.50-4.50$ & $1-5$ \\
Objetivos estratégicos & 42 & 4.2048 & 0.6362 & $2.60-5.00$ & $1-5$ \\
Superficie de la planta en m ${ }^{2}$ & 42 & 4.443 & 8.385 & $100-50.000$ & ----- \\
Año de inicio de actividades & 42 & 1974 & 19.48 & $1929-2002$ & ---- \\
\hline
\end{tabular}


Tabla 3

Relaciones entre factores externos y propósito de uso de SCG

\begin{tabular}{llc}
\hline & \multicolumn{2}{c}{ Propósito de uso de los SCG } \\
\cline { 2 - 3 } & Modelo 1 & Modelo 2 \\
\hline Constante & 1.7987 & 8.4347 \\
Experiencia en mercado local & 0.1261 & 0.1163 \\
Experiencia con la tarea/tecnología & 0.0742 & 0.0470 \\
Inversión (trust) & & 0.1153 \\
Alineación estratégica & & 0.0297 \\
Superficie de la planta & & 0.0002 \\
Años de inicio de actividades & & -0.0035 \\
F (valor de p) & $0.55(0.5799)$ & $0.48(0.8168)$ \\
$\mathrm{R}^{2}$ ajustado & 0.0223 & 0.0820 \\
\hline
\end{tabular}

positiva entre la experiencia con la volatilidad del mercado local y el uso de los SCG para coordinar. La hipótesis $\mathrm{H} 1 \mathrm{~b}$ predecía una relación positiva entre la experiencia con la volatilidad de la tarea y el uso de los SCG para coordinar. Ningún modelo muestra coeficientes estadísticamente significativos, por lo que las hipótesis $\mathrm{H} 1 \mathrm{a}$ y $\mathrm{H} 1 \mathrm{~b}$ pueden ser rechazadas. Interesante es notar que ninguna de las variables de control tiene un rol decisivo en los modelos. En función de estos resultados se afirma que la idea detrás de la hipótesis 1 no es respaldada por los datos empíricos a pesar de haber sido argumentada en estudios de casos (Kattan et al., 2007, Porporato y Garcia, 2011).

Resultados: desempeño y propósito de uso de los sistemas de la contabilidad de gestión

Para evaluar las hipótesis que relacionan el desempeño con los SCG, este trabajo usa la siguiente ecuación de regresión:

Desempeño $=f$ (propósito de uso de los SCG)

La tabla 4 muestra los resultados de 4 regresiones múltiples del tipo OLS que capturan el efecto del propósito de uso de los SCG en el desempeño. Los modelos están en línea con la teoría y capturan bastante bien la realidad con significativos indicadores $\mathrm{F}$ y valores de $\mathrm{R}^{2}$ ajustado en el rango del $9 \%$ al 33\%. La hipótesis $\mathrm{H} 2$ predecía una relación positiva entre el propósito de uso de los SCG y el desempeño. Para el propósito de uso, el modelo 5 muestra un coeficiente estadísticamente significativo, confirmando la idea de que el desempeño mejora si los SCG se usan primordialmente para coordinar. Interesante es notar que de las variables de control solo la alineación estratégica tiene un rol decisivo en todos los modelos. En función de estos resultados se afirma que la hipótesis H2 no puede ser rechazada ya que según lo percibe su gerente, el desempeño de la organización mejora en la medida que los SCG se usan más con el propósito de coordinar (modelos 5 y 6).

El estudio del rol que tiene el propósito de uso de los SCG en el desempeño organizacional ha producido resultados parciales y contradictorios. Estudios de casos sostienen que es un mediador entre factores del entorno y desempeño organizacional (Kattan et al., 2007; Porporato y Garcia, 2011), lo que a priori no se sostiene con los resultados de la tabla 4. También si se considera la literatura en administración de organizaciones que operan en entornos turbulentos como lo son las joint ventures y alianzas estratégicas, se observan resultados dispares. Por ejemplo, hay estudios 
Tabla 4

Relaciones entre desempeño, uso de SCG y factores externos

\begin{tabular}{|c|c|c|c|c|}
\hline \multirow{2}{*}{$\begin{array}{l}\text { Variable Dependiente } \\
\text { Variable Independiente }\end{array}$} & \multicolumn{4}{|c|}{ Desempeño de las industrias cordobesas } \\
\hline & Modelo 3 & Modelo 4 & Modelo 5 & Modelo 6 \\
\hline Constante & 3.3908 & 4.1472 & 2.6603 & 1.4964 \\
\hline Propósito de uso SCG & & & $0.4062 * *$ & $0.3143 * *$ \\
\hline Exper. mercado local & -0.0038 & 0.0577 & -0.0550 & 0.2111 \\
\hline Exper. tarea/industria & -0.0341 & -0.0398 & -0.0642 & -0.0545 \\
\hline Inversión (trust) & & 0.2018 & & 0.1655 \\
\hline Alineación estratégica & & $0.3507 * *$ & & $0.3413 * *$ \\
\hline Superficie de la planta & & 0.0006 & & -0.0005 \\
\hline Años de actividades & & -0.0016 & & -0.0004 \\
\hline $\mathrm{F}$ (valor de p) & $0.03(0.9689)$ & $3.27(0.0117)$ & $2.35(0.0879)$ & $3.93(0.0031)$ \\
\hline $\mathrm{R}^{2}$ ajustado & 0.0496 & 0.2493 & 0.0898 & 0.3333 \\
\hline
\end{tabular}

* Nivel de confianza $10 \%$.

** Nivel de confianza $5 \%$.

que sostienen que existe una relación positiva entre uso de los SCG y el desempeño organizacional (Pangarkar y Klein, 2004), otros que mantienen que la relación es negativa (Boateng y Glaister, 2002), y un tercer grupo que ofrece resultados mixtos al usar regresiones cuadráticas en vez de lineales (Ramaswamy, Gomes y Veliyath, 1998, Porporato, 2009). Los próximos párrafos analizaran ambas discusiones.

\section{Propósito de uso de los sistemas de la contabilidad de gestión como variable mediadora}

Para evaluar la idea que relaciona el desempeño con factores externos, pero mediado o moderado por el propósito de uso de SCG, se emplea el modelo de Baron y Kenny (1986). Se escoge interpretar los SCG como una variable mediadora ya que ello representa mejor la realidad en la que los factores externos afectan el desempeño a través del uso de los SCG. La otra alternativa, considerar los SCG como variables moderadoras, no refleja el rol de los SCG ya que una variable moderadora es aquella que afecta la dirección y/o fuerza de la relación entre los factores externos y el desempeño organizacional. El otro factor que lleva a escoger entre estos enfoques en la modelización es que las variables moderadoras normalmente hacen referencia a intervenciones, mientras que las mediadoras son procesos (fig. 2).

Se afirma que una variable mediadora considera la relación entre las variables independientes (factores externos) y la variable dependiente (el desempeño), y explica cómo los factores externos toman importancia interna en las organizaciones. Los resultados no confirman para las industrias de Córdoba la idea de que los SCG actúan como un mediador entre el desempeño y la experiencia con la volatilidad de los factores externos como la tarea y el entorno. El uso de SCG no es un mediador porque no satisface una de las condiciones y pruebas detalladas por Baron y Kenny (1986): a) las variaciones en los niveles de la variable independiente no afectan considerablemente las variaciones en los mediadores (en nuestro estudio esto es capturado según las hipótesis H1a y H1b donde Propósito de uso de los SCG = $f$ (entorno, tarea), ver tabla 3, modelo 1), b) las variaciones en el mediador afectan considerablemente las variaciones en la variable dependiente (H2 donde Desempeño $=f$ (propósito de uso de los SCG), ver tabla 4, modelo 5), y c) cuando las relaciones antes mencionadas son controladas, la relación entre 


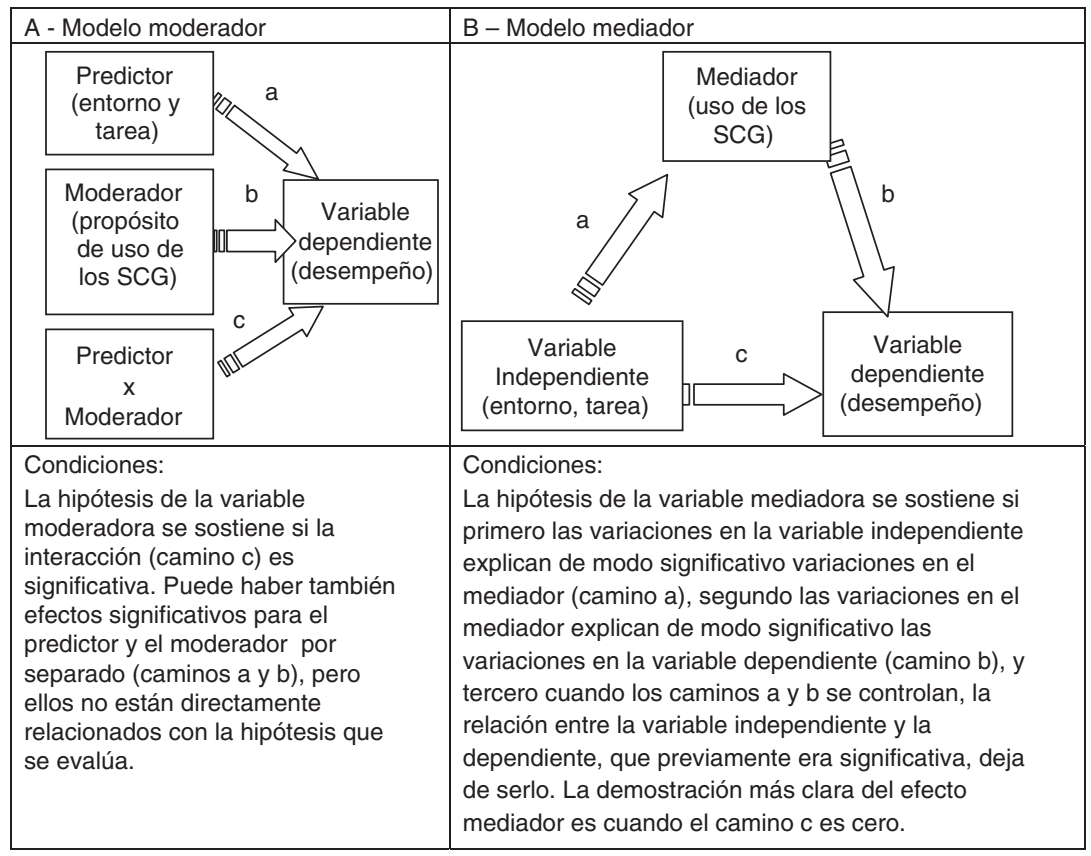

Figura 2. Modelización de variables intervinientes: moderadoras o mediadoras Fuente: Baron y Kenny (1986, pp. 1174 y 1176).

las variables independientes y la dependientes no son significativas (ver tabla 4, modelo 6). El propósito de uso, por definición, tampoco es una variable moderadora por lo que se la debe considerar como otra variable independiente tal como se hizo en los modelos 5 y 6 de la tabla 4.

\section{Propósito de uso de los sistemas de la contabilidad de gestión: comportamiento cuadrático respecto el desempeño}

El análisis complementario que resta es determinar si una relación cuadrática es significativa. La contribución de usar modelos cuadráticos para modelar la relación entre propósito de uso de los SCG y desempeño organizacional es poder determinar hasta qué nivel se usan los SCG con el propósito de coordinar (relación positiva) y a partir de qué momento se empiezan a utilizar para controlar. Estudios previos muestran que en joint ventures el referido punto ocurre entre $2.89 \mathrm{y}$ 2.96 (escala: 1 con SCG usados para controlar y 5 para coordinar), indicando que el desempeño organizacional mejora si los SCG se usan inicialmente para coordinar, pero a partir de cierto punto del desempeño organizacional (2.43 y 3.77 escala: 5 es el máximo), el desempeño organizacional mejora si los SCG empiezan a ser empleados más bien para controlar (Porporato, 2009).

Para efectuar este análisis primero se calculan los coeficientes no estandarizados en una regresión cuadrática, y segundo el punto de inflexión se determina usando derivadas parciales (tabla 5). Los resultados muestran que la relación entre desempeño y propósito de uso de los SCG se puede modelar también como una relación cuadrática y que el punto de inflexión (2,7913 para propósito de uso SCG y 3,4284 para desempeño) es menor que el documentado en joint ventures con el mismo modelo $(2,8891$ y 3,7756$)$. Este resultado indica que en industrias cordobesas el desempeño mejora si inicialmente los SCG se usan para coordinar, pero para seguir mejorando 
Tabla 5

Propósito de uso de los SCG: regresión cuadrática y punto de inflexión

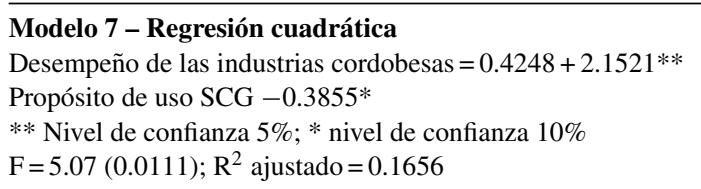

Cálculo del punto de Inflexión

El punto de inflexión se calcula obteniendo la derivada parcial de la regresión del modelo 7 respecto la variable

Propósito de uso SCG:

Desempeño = $2.1521-2(0.3855$ Propósito de uso $)$

Propósito de uso

\author{
Desempeño $=2.1521-0.771$ Propósito de uso) \\ Propósito de uso \\ Para obtener el punto de inflexión, la ecuación obtenida se iguala a cero y se resuelve para Propósito de uso: \\ 0.771 Propósito de uso $=2.1521$ \\ Propósito de uso = 2.1521/0.771; Propósito de uso = 2.7913 \\ Reemplazando en la ecuación del modelo 7 tenemos: \\ Desempeño industrias cordobesas $=0.4248+2.1521 \times 2.7913-0.3855 \times 2.7913^{2}$ \\ Desempeño industrias cordobesas $=3.4284$
}

el desempeño se deben incluir mecanismos de control a niveles más bajos de desempeño que en el caso de las joint ventures. La literatura de administración considera las joint ventures como organizaciones operando en condiciones muy turbulentas con bajos niveles de éxito o supervivencia, sin embargo, por los resultados aquí obtenidos, pareciera ser que las industrias cordobesas operan en condiciones más turbulentas que las joint ventures.

\title{
Conclusiones
}

Varios estudios han sugerido que los mecanismos de la contabilidad de gestión afectan el desempeño de las empresas en entornos turbulentos, pero aún no hay convergencia en la explicación de si el impacto es positivo o negativo. Este estudio, basándose en la teoría de los costos de transacción se enfocó en determinar el impacto sobre el desempeño organizacional que tiene el propósito de uso de los SCG que los diferencia según como sean usados, para coordinar entre agentes y principales o para que el principal controle al agente.

El marco conceptual de este trabajo se fundamenta en 2 teorías. Costos de transacción aplicados a la contabilidad explica que hay 2 maneras de mirar el rol de los SCG, ya sean instrumentos de control para reducir la divergencia de los intereses entre los miembros de la organización (control), ya como la infraestructura de información que los miembros de la organización utilizan para resolver la incertidumbre que enfrentan en sus decisiones (coordinación). Hay que destacar que el concepto de incertidumbre aquí empleado es el definido por Galbraith (1973) y usado consistentemente en estudios posteriores en contabilidad de gestión. La segunda es la teoría de la contingencia, y es la que justifica el modelo de relaciones entre variables independientes y dependientes. La variable dependiente es el desempeño organizacional mientras que las variables independientes son factores contingentes tales como el entorno y tarea. De las varias relaciones que se emplearon para modelizar el propósito de uso de los SCG se infiere, contrariamente 
a los resultados de estudios de casos (Kattan et al., 2007; Porporato y Garcia, 2011), que el propósito de uso es más bien una variable independiente pero no mediadora o moderadora según la terminología empleada por Baron y Kenny (1986).

El propósito de este trabajo ha sido contribuir al entendimiento del rol que desempeñan los SCG en el éxito o fracaso de las organizaciones en entornos turbulentos, particularmente en Latinoamérica. Con respecto al uso de los SCG, la percepción de los gerentes permite agregar contenido a la literatura sobre el tema. Las conclusiones son de naturaleza parcial porque se ha empleado un modelo contingente para comprobar las relaciones existentes entre factores, propósito de uso de los SCG y desempeño organizacional. Sin embargo, la principal relación que se ha podido establecer es que el desempeño mejora mediante un uso de la contabilidad orientado más a coordinar que a controlar, y que los factores del entorno o tarea no afectan el desempeño pero que el mismo sí mejora cuando aumenta el interés en la empresa (alineación estratégica y nivel de inversión). Interesante es notar que la relación positiva entre desempeño y uso de los SCG para coordinar tiene un límite a partir del cual el desempeño mejora solo si el balance en el uso de los SCG se orienta a controlar.

Este trabajo identifica para las organizaciones que operan en entornos turbulentos el impacto en el desempeño de los mecanismos de la contabilidad de gestión según su propósito de uso (coordinar o controlar). Con respecto a la contribución de los SCG al éxito en empresas manufactureras de Córdoba, se indica un efecto positivo cuando se usan para coordinar. Al buscar explicaciones alternativas, se encontró que el desempeño mejora mientras mayor sea la alineación de la estrategia y el compromiso de los socios. La idea de que los SCG se utilizan para reducir la incertidumbre generada por factores que no se perciben como influenciables por las acciones de la gerencia es concordante con lo sugerido en la literatura (Porporato y Garcia, 2011).

Este trabajo indica que en entornos turbulentos el uso de la información de los SCG con propósitos de coordinación mejora el desempeño hasta un cierto nivel, a partir del cual son necesarios mecanismos de control. Sin descontar las limitaciones a la validez externa de este estudio, se muestra que para mejorar la gestión del desempeño organizacional de entidades que operan en ambientes turbulentos, sus directivos deben pensar y diseñar los sistemas de control de gestión como un mecanismo para compartir información relevante (solo sobre factores que se perciban gestionables por los miembros de la organización) para la toma de decisiones entre todas las partes vinculadas. Compartir información aumenta la confianza o compromiso de los socios y hace más evidente la estrategia de la organización. Sin embargo, a medida que la organización crece, las necesidades de información crecen así como la cantidad de datos disponibles, lo que puede generar conductas no deseables entre sus participantes (incentivos a maximizar el beneficio personal minimizando el esfuerzo contribuido a la organización). Para evitar o al menos minimizar las conductas no deseables, se deben incluir sistemas de control de gestión dedicados a controlar tales como la medición del desempeño personal, la evaluación de desempeño por áreas y los sistemas de compensación e incentivos.

Este trabajo cuenta con varias limitaciones, la principal a destacar es el tamaño de la muestra que afecta la consistencia de los resultados. El cuestionario desarrollado y adaptado para capturar el impacto del propósito de uso de los SCG parece ser firme y puede ser usado en investigaciones futuras. Deberían ser diseñados próximos estudios para rechazar o no la idea de que los SCG usados principalmente para coordinar mejoran el desempeño de las organizaciones en entornos turbulentos; en especial poder mostrar de un modo inequívoco el efecto de las diferencias del propósito de uso de los SCG sobre el desempeño organizacional y sus relaciones con factores contingentes. 


\section{Apéndice. Medición de las variables en el instrumento de la encuesta y estimación de factores}

\section{Variable dependiente: Desempeño percibido-Industrias cordobesas}

Ítems en el cuestionario

Pregunta 2: Cómo calificaría el DESEMPEÑO de su empresa en términos de:

\begin{tabular}{|c|c|c|c|c|c|}
\hline Evaluación de desempeño & Muy bajo & Bajo & Normal & Alto & Muy alto \\
\hline Desempeño real comparado con las estimaciones iniciales & $\square$ & $\square$ & $\square$ & $\square$ & $\square$ \\
\hline Operar acorde las normas, leyes y regulaciones & $\square$ & $\square$ & $\square$ & $\square$ & $\square$ \\
\hline Cumplir con los plazos de entrega y trabajo requeridos & $\square$ & $\square$ & $\square$ & $\square$ & $\square$ \\
\hline Ser rentable (operar acorde presupuestos y costos) & $\square$ & $\square$ & $\square$ & $\square$ & $\square$ \\
\hline Satisfacer las expectativas de los clientes & $\square$ & $\square$ & $\square$ & $\square$ & $\square$ \\
\hline Producir calidad usando tecnología actual & $\square$ & $\square$ & $\square$ & $\square$ & $\square$ \\
\hline Evolución de la cuota de mercado & $\square$ & $\square$ & $\square$ & $\square$ & $\square$ \\
\hline Indicadores financieros (montos y ratios) & $\square$ & $\square$ & $\square$ & $\square$ & $\square$ \\
\hline Indicadores operativos (defectos por millón) & $\square$ & $\square$ & $\square$ & $\square$ & $\square$ \\
\hline Indicadores subjetivos (evaluación de empleados) & $\square$ & $\square$ & $\square$ & $\square$ & $\square$ \\
\hline Evaluación general del desempeño de su empresa & $\square$ & $\square$ & $\square$ & $\square$ & $\square$ \\
\hline Nivel de satisfacción de los dueños de la empresa & $\square$ & $\square$ & $\square$ & $\square$ & $\square$ \\
\hline Nivel de satisfacción de los empleados de la empresa & $\square$ & $\square$ & $\square$ & $\square$ & $\square$ \\
\hline
\end{tabular}

Concepto empleado en la modelización

\begin{tabular}{|c|c|c|c|c|}
\hline Factor: Desempeño organizacional & Media (DE) & $\begin{array}{l}\text { Rango teórico } \\
\text { 1.Muy bajo } \\
\text { 5. Muy alto }\end{array}$ & $\begin{array}{l}\text { Rango actual } \\
\text { 1.Muy bajo } \\
\text { 5.Muy alto }\end{array}$ & $\begin{array}{l}\text { Peso del } \\
\text { primer } \\
\text { factor }\end{array}$ \\
\hline $\begin{array}{l}\text { Desempeño real comparado con } \\
\text { estimaciones iniciales }\end{array}$ & $3.177(0.833)$ & $1-5$ & $2-5$ & 0.63754 \\
\hline $\begin{array}{l}\text { Operar acorde las normas, leyes y } \\
\text { regulaciones }\end{array}$ & $3.844(0.796)$ & $1-5$ & $2-5$ & 0.48790 \\
\hline $\begin{array}{l}\text { Cumplir con los plazos de entrega y } \\
\text { trabajo requeridos }\end{array}$ & $3.444(0.989)$ & $1-5$ & $1-5$ & 0.67636 \\
\hline $\begin{array}{l}\text { Ser rentable (operar acorde presupuestos y } \\
\text { costos) }\end{array}$ & $3.111(0.910)$ & $1-5$ & $1-5$ & 0.64947 \\
\hline Satisfacer las expectativas de los clientes & $3.777(0.735)$ & $1-5$ & $2-5$ & 0.54763 \\
\hline Producir calidad usando tecnología actual & $3.466(0.814)$ & $1-5$ & $1-5$ & 0.72015 \\
\hline Evolución de la cuota de mercado & $3.088(0.763)$ & $1-5$ & $1-5$ & 0.46454 \\
\hline Indicadores financieros (montos y ratios) & $3.022(0.690)$ & $1-5$ & $1-5$ & 0.66975 \\
\hline $\begin{array}{l}\text { Indicadores operativos (defectos por } \\
\text { millón) }\end{array}$ & $2.755(0.980)$ & $1-5$ & $1-5$ & 0.45510 \\
\hline $\begin{array}{l}\text { Indicadores subjetivos (evaluación de } \\
\text { empleados) }\end{array}$ & $3.111(0.745)$ & $1-5$ & $1-5$ & 0.60627 \\
\hline $\begin{array}{l}\text { Evaluación general del desempeño de su } \\
\text { empresa }\end{array}$ & $3.177(0.716)$ & $1-5$ & $2-5$ & 0.90594 \\
\hline $\begin{array}{l}\text { Nivel de satisfacción de los dueños de la } \\
\text { empresa }\end{array}$ & $3.155(0.796)$ & $1-5$ & $2-5$ & 078570 \\
\hline $\begin{array}{l}\text { Nivel de satisfacción de los empleados de } \\
\text { la empresa }\end{array}$ & $3.133(0.726)$ & $1-5$ & $2-5$ & 051173 \\
\hline Variación explicada & & & & 0.6743 \\
\hline Valor propio & & & & 5.28123 \\
\hline Alfa de Cronbach & & & & 0.8818 \\
\hline
\end{tabular}




\section{Propósito de uso de los sistemas de la contabilidad de gestión}

Ítems en el cuestionario

Pregunta 21: Debajo se describen 2 USOS EXTREMOS DE LA INFORMACIÓN del sistema de gestión que normalmente usa.

a) La información sirve para CONTROLAR pero no es discutida excepto cuando los resultados son diferentes de lo planeado.

b) La información es constantemente empleada para COORDINAR, suele ser el tema más importante de su conversación.

Marque con una X cómo se usa cada tipo de información:

\begin{tabular}{llllll}
\hline & $\begin{array}{l}\text { Solo para } \\
\text { CONTROLAR }\end{array}$ & $\begin{array}{l}\text { Principalmente } \\
\text { para controlar }\end{array}$ & $\begin{array}{l}\text { Para controlar } \\
\text { y coordinar }\end{array}$ & $\begin{array}{l}\text { Principalmente } \\
\text { para coordinar }\end{array}$ & $\begin{array}{l}\text { Solo para } \\
\text { COORDINAR }\end{array}$ \\
\hline $\begin{array}{l}\text { Información sobre leyes y } \\
\text { regulaciones }\end{array}$ & $\square$ & $\square$ & $\square$ & $\square$ & $\square$ \\
$\begin{array}{l}\text { Información sobre plazos de } \\
\text { entrega }\end{array}$ & $\square$ & $\square$ & $\square$ & $\square$ & $\square$ \\
$\begin{array}{l}\text { Información de resultados } \\
\quad \text { financieros }\end{array}$ & $\square$ & $\square$ & $\square$ & $\square$ & $\square$ \\
$\begin{array}{l}\text { Información sobre } \\
\text { expectativas de clientes }\end{array}$ & $\square$ & $\square$ & $\square$ & $\square$ & $\square$ \\
$\begin{array}{l}\text { Información sobre calidad y } \\
\text { tecnología }\end{array}$ & $\square$ & $\square$ & $\square$ & $\square$ & $\square$ \\
\hline
\end{tabular}

Pregunta 24: Debajo se describen 2 USOS EXTREMOS DE LOS REPORTES del sistema de gestión que usted normalmente usa.

a) Los reportes sirven para CONTROLAR pero no son discutidos excepto cuando los resultados son diferentes de lo planeado.

b) Los reportes son constantemente empleados para COORDINAR, suelen ser el tema más importante de su conversación.

Marque con una X qué tipo de información se usa en cada caso:

\begin{tabular}{llllll}
\hline & $\begin{array}{l}\text { Solo para } \\
\text { CONTROLAR }\end{array}$ & $\begin{array}{l}\text { Principalmente } \\
\text { para controlar }\end{array}$ & $\begin{array}{l}\text { Para controlar y } \\
\text { coordinar }\end{array}$ & $\begin{array}{l}\text { Principalmente } \\
\text { para coordinar }\end{array}$ & $\begin{array}{l}\text { Solo para } \\
\text { COORDINAR }\end{array}$ \\
\hline Planeamiento estratégico & $\square$ & $\square$ & $\square$ & $\square$ & $\square$ \\
Presupuestación & $\square$ & $\square$ & $\square$ & $\square$ & $\square$ \\
Planeamiento operativo & $\square$ & $\square$ & $\square$ & $\square$ & $\square$ \\
$\begin{array}{l}\text { Medición del desempeño } \\
\quad \text { personal }\end{array}$ & $\square$ & $\square$ & $\square$ & $\square$ & $\square$ \\
$\begin{array}{l}\text { Evaluación de desempeño } \\
\quad \text { por áreas }\end{array}$ & $\square$ & $\square$ & $\square$ & $\square$ & $\square$ \\
$\begin{array}{l}\text { Sistemas de } \\
\quad \text { compensación e }\end{array}$ & $\square$ & $\square$ & $\square$ & $\square$ & $\square$ \\
$\quad$ incentivos & & & &
\end{tabular}




\begin{tabular}{|c|c|c|c|c|c|}
\hline & $\begin{array}{l}\text { Solo para } \\
\text { CONTROLAR }\end{array}$ & $\begin{array}{l}\text { Principalmente } \\
\text { para controlar }\end{array}$ & $\begin{array}{l}\text { Para controlar y } \\
\text { coordinar }\end{array}$ & $\begin{array}{l}\text { Principalmente } \\
\text { para coordinar }\end{array}$ & $\begin{array}{l}\text { Solo para } \\
\text { COORDINAR }\end{array}$ \\
\hline Medición de costos & $\square$ & $\square$ & $\square$ & $\square$ & $\square$ \\
\hline Sistemas de costos & $\square$ & $\square$ & $\square$ & $\square$ & $\square$ \\
\hline $\begin{array}{l}\text { Procedimientos de } \\
\text { asignación de costos }\end{array}$ & $\square$ & $\square$ & $\square$ & $\square$ & $\square$ \\
\hline $\begin{array}{l}\text { Delimitación de centros } \\
\text { de responsabilidad }\end{array}$ & $\square$ & $\square$ & $\square$ & $\square$ & $\square$ \\
\hline $\begin{array}{l}\text { Precios de transferencia } \\
\quad \text { (internos) }\end{array}$ & $\square$ & $\square$ & $\square$ & $\square$ & $\square$ \\
\hline $\begin{array}{l}\text { Determinación de precios } \\
\text { de venta (externos) }\end{array}$ & $\square$ & $\square$ & $\square$ & $\square$ & $\square$ \\
\hline $\begin{array}{l}\text { Cambios en la } \\
\text { participación de los } \\
\text { dueños }\end{array}$ & $\square$ & $\square$ & $\square$ & $\square$ & $\square$ \\
\hline Designación de gerentes & $\square$ & $\square$ & $\square$ & $\square$ & $\square$ \\
\hline $\begin{array}{l}\text { Designación del auditor } \\
\text { externo }\end{array}$ & $\square$ & $\square$ & $\square$ & $\square$ & $\square$ \\
\hline
\end{tabular}

\section{Concepto empleado en la modelización}

Factor: Propósito de uso

Ítems en el cuestionario

Media (DE)

Rango teórico
1: Control
5: Coordinación

Rango actual

Peso en el

5: Coordinación

1: Control

5: Coordinación

primer

factor

\begin{tabular}{llccc}
\hline \multicolumn{2}{l}{ Tipos de información de la contabilidad de gestión } & & & \\
Información sobre leyes y regulaciones & $2.355(1.0478)$ & $1-5$ & $1-5$ & 0.15459 \\
Información sobre plazos de entrega & $3.177(0.747)$ & $1-5$ & $1-5$ & 0.09775 \\
Información de resultados financieros & $2.911(0.820)$ & $1-5$ & $1-5$ & 0.01439 \\
Información sobre expectativas de clientes & $3.355(0.856)$ & $1-5$ & $1-5$ & 0.40977 \\
Información sobre calidad y tecnología & $2.955(1.021)$ & $1-5$ & $1-5$ & 0.31022 \\
Sistemas componentes de la contabilidad de gestión & $3.288(1.036)$ & $1-5$ & $1-5$ & -0.05118 \\
Planeamiento estratégico & $3.133(0.943)$ & $1-5$ & $1-5$ & 048299 \\
Presupuestación & $3.200(0.990)$ & $1-5$ & $1-5$ & 0.69523 \\
Planeamiento operativo & $2.511(1.014)$ & $1-5$ & $1-5$ & 0.78141 \\
Medición del desempeño personal & $2.622(1.028)$ & $1-5$ & $1-5$ & 0.56915 \\
Evaluación de desempeño por áreas & $2.600(1.053)$ & $1-5$ & $1-5$ & 0.57863 \\
Sistemas de compensación e incentivos & $2.644(0.773)$ & $1-5$ & $1-4$ & 0.48330 \\
Medición de costos & $2.688(0.820)$ & $1-5$ & $1-5$ & 0.39263 \\
Sistemas de costos & $2.822(0.886)$ & $1-5$ & $1-5$ & 0.63869 \\
Procedimientos de asignación de costos & $2.688(1.124)$ & $1-5$ & $1-5$ & 0.38278 \\
Delimitación de centros de responsabilidad & $2.688(0.924)$ & $1-5$ & $1-5$ & 0.30386 \\
Precios de transferencia (internos) & $3.000(0.639)$ & $1-5$ & $1-5$ & 0.59806 \\
Determinación de precios de venta (externos) & & 0.55593 \\
Cambios en la participación de los dueños & $2.355(1.367)$ & $1-5$ & $1-5$ & 0.56471 \\
Designación de gerentes & $2.444(1.357)$ & $1-5$ & $1-5$ & 0.15459 \\
Designación del auditor externo & $2.333(1.224)$ & $1-5$ & $1-5$ & \\
\hline
\end{tabular}

Variación explicada 


\section{Variables independientes: Factores externos}

Ítems en el cuestionario

Pregunta 3: Evalúe el nivel de cambios experimentados en cada uno de los FACTORES que afectan el desempeño de su empresa:

\begin{tabular}{llllll}
\hline Volatilidad & Muy baja & Baja & Normal & Alta & Muy alta \\
\hline Proceso de producción (manufactura) & $\square$ & $\square$ & $\square$ & $\square$ & $\square$ \\
Tecnología de producción & $\square$ & $\square$ & $\square$ & $\square$ & $\square$ \\
Clientes/mercado objetivo & $\square$ & $\square$ & $\square$ & $\square$ & $\square$ \\
Competidores & $\square$ & $\square$ & $\square$ & $\square$ & $\square$ \\
Empresas similares (tamaño, clientes o localización) & $\square$ & $\square$ & $\square$ & $\square$ & $\square$ \\
Requisitos exigidos por el gobierno & $\square$ & $\square$ & $\square$ & $\square$ & $\square$ \\
\hline
\end{tabular}

Pregunta 30: Indique el grado de CAMBIO en la COMPETENCIA que sus productos o servicios han experimentado en los últimos 5 años:

$\square$ Disminución importante $\square$ Disminución leve $\square$ Sin cambio $\square$ Aumento leve $\square$ Aumento importante

Pregunta 31: Indique el grado en el que la competencia ha CAMBIADO la organización del sistema de CONTROL DE GESTIÓN:

$\square$ Sin cambios $\square$ C. mínimos $\square$ C. moderados $\square$ C. importantes $\square$ Cambios muy importantes

Pregunta 33: Indique el grado de INTENSIDAD DE LA COMPETENCIA percibida por su empresa respecto a las siguientes variables:

\begin{tabular}{llllll}
\hline & Muy baja & Baja & Normal & Alta & Muy alta \\
\hline Ventas y distribución & $\square$ & $\square$ & $\square$ & $\square$ & $\square$ \\
Calidad & $\square$ & $\square$ & $\square$ & $\square$ & $\square$ \\
Variedad de productos & $\square$ & $\square$ & $\square$ & $\square$ & $\square$ \\
Otros (por favor, explicar): & $\square$ & $\square$ & $\square$ & $\square$ & $\square$ \\
\hline
\end{tabular}

Pregunta 35: Indique en qué medida los siguientes CAMBIOS TECNOLÓGICOS han afectado el sistema de CONTROL de GESTIÓN:

\begin{tabular}{llllll}
\hline & Muy bajo & Bajo & Normal & Alto & Muy alto \\
\hline Automatización & $\square$ & $\square$ & $\square$ & $\square$ & $\square$ \\
Ciclos productivos cortos & $\square$ & $\square$ & $\square$ & $\square$ & $\square$ \\
Aumento en costos indirectos & $\square$ & $\square$ & $\square$ & $\square$ & $\square$ \\
Requerimientos de calidad & $\square$ & $\square$ & $\square$ & $\square$ & $\square$ \\
Otros (por favor, explicar): & $\square$ & $\square$ & $\square$ & $\square$ & $\square$ \\
\hline
\end{tabular}

Pregunta 36: Indique la importancia de cada uno de los siguientes factores que IMPIDEN CAMBIOS en el sistema de control de gestión:

\begin{tabular}{|c|c|c|c|c|c|}
\hline & Muy baja & Baja & Normal & Alta & Muy alta \\
\hline Falta de personal adecuado (contables o administrativos) & $\square$ & $\square$ & $\square$ & $\square$ & $\square$ \\
\hline Falta de adecuados recursos informáticos & $\square$ & $\square$ & $\square$ & $\square$ & $\square$ \\
\hline Inercia & $\square$ & $\square$ & $\square$ & $\square$ & $\square$ \\
\hline
\end{tabular}




\begin{tabular}{|c|c|c|c|c|c|}
\hline & Muy baja & Baja & Normal & Alta & Muy alta \\
\hline Comunicación limitada entre los gerentes y el personal & $\square$ & $\square$ & $\square$ & $\square$ & $\square$ \\
\hline Falta de autoridad del experto contable o administrativo & $\square$ & $\square$ & $\square$ & $\square$ & $\square$ \\
\hline Necesidad de cumplir con lo requerido por la ley & $\square$ & $\square$ & $\square$ & $\square$ & $\square$ \\
\hline Otros (por favor, explicar): & $\square$ & $\square$ & $\square$ & $\square$ & $\square$ \\
\hline
\end{tabular}

Conceptos empleados en la modelización

\begin{tabular}{|c|c|c|c|}
\hline \multicolumn{4}{|c|}{ Concepto: Volatilidad percibida-Industrias cordobesas } \\
\hline $\begin{array}{l}\text { Factor: Volatilidad del entorno } \\
\text { Ítems en el cuestionario }\end{array}$ & $\begin{array}{l}\text { Peso del primer } \\
\text { factor }\end{array}$ & $\begin{array}{l}\text { Factor: Volatilidad de la tarea } \\
\text { Ítems en el cuestionario }\end{array}$ & $\begin{array}{l}\text { Peso del primer } \\
\text { factor }\end{array}$ \\
\hline Grado de cambio de la competencia & 0.40784 & $\begin{array}{l}\text { Reacciones a cambios en: } \\
\text { Automatización }\end{array}$ & 0.52891 \\
\hline $\begin{array}{l}\text { Reacciones al cambio en la } \\
\text { competencia }\end{array}$ & 0.65219 & Ciclos productivos cortos & 0.61012 \\
\hline $\begin{array}{l}\text { Intensidad en la competencia: } \\
\text { Ventas y distribución }\end{array}$ & 0.58491 & $\begin{array}{l}\text { Aumento de los costos } \\
\text { indirectos }\end{array}$ & 0.61800 \\
\hline Calidad & 0.62686 & Requerimientos de calidad & 0.67126 \\
\hline Variedad de productos & 054942 & $\begin{array}{l}\text { Factores que impiden } \\
\text { cambios: } \\
\text { Falta de personal }\end{array}$ & 0.61046 \\
\hline $\begin{array}{l}\text { Volatilidad de cliente/mercado } \\
\text { objetivo }\end{array}$ & 0.42042 & Falta de recursos informáticos & 0.49911 \\
\hline Volatilidad de competidores & 050824 & Inercia & 0.59470 \\
\hline Volatilidad de empresas similares & 052648 & Comunicación limitada & 067510 \\
\hline \multirow{4}{*}{$\begin{array}{l}\text { Volatilidad de los requisitos del } \\
\text { gobierno }\end{array}$} & 0.19366 & Falta de autoridad & 0.66434 \\
\hline & & Requisitos legales & 0.45825 \\
\hline & & $\begin{array}{l}\text { Volatilidad del proceso de } \\
\text { producción }\end{array}$ & 0.63093 \\
\hline & & $\begin{array}{l}\text { Volatilidad de tecnología de } \\
\text { producción }\end{array}$ & 0.31122 \\
\hline Variación explicada & 0.6085 & & 0.5743 \\
\hline Valor propio & 2.37836 & & 4.06197 \\
\hline Alfa de Cronbach & 0.7196 & & 0.8398 \\
\hline
\end{tabular}

\section{Variables de control}

Ítems en el cuestionario

Pregunta 4: Cómo calificaría en términos de importancia cada uno de los siguientes OBJETIVOS ESTRATÉGICOS tanto para los gerentes como para los dueños de su empresa (si quien responde además de gerente es uno de los dueños, por favor solo evalúe uno):

\begin{tabular}{llllll}
\hline & Mínima & Baja & Normal & Alta & Muy alta \\
\hline Ser rentable (operar acorde a presupuestos y costos) & $\square$ & $\square$ & $\square$ & $\square$ & $\square$ \\
Cumplir con los plazos de entrega/trabajo requeridos & $\square$ & $\square$ & $\square$ & $\square$ & $\square$ \\
Operar acorde a las normas, leyes y regulaciones & $\square$ & $\square$ & $\square$ & $\square$ & $\square$ \\
Satisfacer las expectativas de los clientes & $\square$ & $\square$ & $\square$ & $\square$ & $\square$ \\
Producir acorde a especificaciones & $\square$ & $\square$ & $\square$ & $\square$ & $\square$ \\
\hline
\end{tabular}


Pregunta 5: Indique cómo calificaría la INVERSIÓN efectuada en cada uno de los siguientes ítems:

\begin{tabular}{|c|c|c|c|c|c|}
\hline Inversión & Muy baja & Baja & Normal & Alta & Muy alta \\
\hline Personal (empleados) & $\square$ & $\square$ & $\square$ & $\square$ & $\square$ \\
\hline Instalaciones (edificios y maquinarias) & $\square$ & $\square$ & $\square$ & $\square$ & $\square$ \\
\hline Inversión total (medida monetariamente) & $\square$ & $\square$ & $\square$ & $\square$ & $\square$ \\
\hline Importancia del negocio (medido subjetivamente) & $\square$ & $\square$ & $\square$ & $\square$ & $\square$ \\
\hline
\end{tabular}

\section{Conceptos empleados en la modelización}

\begin{tabular}{lcccc}
\hline \multicolumn{4}{c}{ Factor: Importancia de los objetivos estratégicos } \\
\hline Ítems en el cuestionario & Media & Rango teórico & Rango actual & $\begin{array}{l}\text { Peso del } \\
\text { primer factor }\end{array}$ \\
\hline Ser rentable (operar acorde a presupuestos y costos) & 4.044 & $1-5$ & $2-5$ & 0.74225 \\
Cumplir con los plazos de entrega/trabajo requeridos & 4.289 & $1-5$ & $3-5$ & 0.89315 \\
Operar acorde a las normas, leyes y regulaciones & 4.067 & $1-5$ & $2-5$ & 0.85635 \\
Satisfacer los requerimientos de los clientes & 4.444 & $1-5$ & $3-5$ & 0.50738 \\
Producir acorde a especificaciones & 4.222 & $1-5$ & $3-5$ & 0.46554 \\
Variación explicada & & 0.74894 & & \\
Valor propio & & 2.55614 & & \\
Alfa de Cronbach & & 0.7912 & & \\
\hline
\end{tabular}

Factor: Inversión en la empresa (confianza)

Ítems en el cuestionario

Peso del primer Variación Valor propio Alfa de

factor explicada Cronbach

Inversión en personal (empleados)

Inversión en instalaciones (edificios y maquinarias)

Inversión total (medida monetariamente)

Importancia del negocio (medida subjetivamente)
0.44487

0.77469

0.83207

0.48453

$0.7374 \quad 1.72518$

0.71449

\section{Referencias}

Anthony, R. N. (1965). The management control function. Boston: Harvard Business School., reprint 1988.

Anthony, R. N. y Govindarajan, V. (2007). Management control systems (12th ed). Boston, Mass: McGraw-Hill/Irwin.

Ariño, A. (2003). Measures of strategic alliance performance: An analysis of construct validity. Journal of International Business Studies, 34, 66-79.

Baron, R. y Kenny, D. (1986). The moderator-mediator variable distinction in social psychological research: Conceptual, strategic, and statistical consideration. Journal of Personality and Social Psychology, 51(6), 1173-1182.

Boateng, A. y Glaister, K. (2002). Performance of international joint ventures: Evidence for West Africa. International Business Review, 11(5), 523-541.

Brownell, P. (1985). Budgetary systems and the control of functionally differentiated organizational activities. Journal of Accounting Research, 23(2), 502-512.

Camillus, J. y Datta, D. (1991). Managing strategic issues in a turbulent environment. Long Range Planning, 24(2), 67-74.

Carmines, E. y Zeller, R. (1979). Reliability and validity assessment. Twelfth printing 1989, Sage University Papers, Series on Quantitative Applications in the Social Sciences. Newbury Park: Sage Publ.

Chenhall, M. (2007). Contingency theory, chapter 6. En C. Chapman, A. Hopwood, y M. Shields (Eds.), Handbook of management accounting research. Oxford, U.K: Elsevier. 
Chenhall, R. H. y Morris, D. (1986). The impact of structure, environment, and interdependence on the perceived usefulness of management accounting systems. The Accounting Review, 61(1), 16-35.

Cronbach, L. J. (1951). Coefficient alpha and the internal structure of tests. Psychometrika, 16(3), $297-334$.

Davila, A. (2000). An empirical study on the drivers of management control systems' design in new product development. Accounting, Organizations and Society, 25(4/5), 383-409.

Dekker, H. (2004). Control of inter-organizational relationships: Evidence on appropriation concerns and coordination requirements. Accounting, Organizations and Society, 29(1), 27-50.

Demski, J. (2008). Managerial uses of accounting information. Springer series in accounting scholarship: Volume 4 (2nd ed.). Gainesville, FL: Springer Science+Business Media.

Demski, J., Fellingham, J., Ijiri, Y., Sunder, S., Glover, J. y Jinghong Liang, P. (2002). Some thoughts on the intellectual foundations of accounting. Accounting Horizons, 16(2), 157-169.

Dillman, D. A. (2009). Internet, mail, and mixed-mode surveys: The tailored design method (3rd ed). Hoboken, NJ: John Wiley Co.

Drazin, R. y van de Ven, A. (1985). Alternative forms of fit in contingency theory. Administrative Science Quarterly, 30(4), 514-539.

Galbraith, J. (1973). Designing complex organizations. Organization development series. Reading, MA: Addison-Wesley.

Gordon, G. y Narayanan, V. (1984). Management accounting systems, perceived environmental uncertainty and organization structure: An empirical investigation. Accounting, Organizations and Society, 9(1), 33-47.

Govindarajan, V. (1988). A contingency approach to strategy implementation at the business-unit level: Integrating administrative mechanisms with strategy. Academy of Management Journal, 31(4), 828-853.

Grant, R. (2003). Strategic planning in a turbulent environment: Evidence from the oil majors. Strategic Management Journal, 24(6), 491-517.

Groot, T. y Merchant, K. (2000). Control of international joint ventures. Accounting, Organizations and Society, 25(6), 579-607.

Hayes, D. (1977). The contingency theory of managerial accounting. The Accounting Review, 52(1), 22-39.

Hofstede, G. (1981). Management control of public and non-for-profit activities. Accounting, Organizations and Society, 6(3), 193-211.

Kaplan, R. (1998). Advanced management accounting (3rd ed). Englewood Cliffs, NJ: Prentice Hall, Inc.

Kattan, F., Pike, R. y Tayles, M. (2007). Reliance of management accounting under environmental uncertainty: The case of Palestine. Journal of Accounting \& Organizational Change, 3(3), 227-249.

Khandwalla. (1972). The effect of different types of competition on the use of management controls. Journal of Accounting Research, 10(2), 275-285.

Kren, R. y Liao, W. (1988). The role of accounting information in the control of organizations: A review of the evidence. Journal of Accounting Literature, 7, 280-309.

McGrath, M. (1995). Product strategy for high-technology companies. New York: Irwin.

Merchant, K. y van der Stede. (2011). Management control systems: Performance measurement, evaluation and incentives (3rd ed). Prentice Hall.

Pangarkar, N. y Klein, S. (2004). The impact of control on international joint venture performance: A contingent approach. Journal of International Marketing, 12(3), 86-108.

Porporato, M. (2009). Impact of formal management control systems on international joint venture performance. Journal for Global Business Advancement, special issue on global accounting issues, 2(1/2), 142-172.

Porporato, M. y Garcia, N. (2011). Sistemas de control de gestión: un estudio exploratorio de su efecto sobre el desempeño organizacional. Academia Revista Latinoamericana de Administración, 47, 61-77.

Ramaswamy, K., Gomes, L. y Veliyath, R. (1998). The performance correlates of ownership control: A study of U.S. and European MNE joint ventures in India. International Business Review, 7(4), 423-441.

Ronen, J. y McKinney, G. (1970). Transfer pricing for divisional autonomy. Journal of Accounting Research, 8(1), 99-112.

Shields, M. (1997). Research in management accounting by North Americans in the 1990s. Journal of Management Accounting Research, 9, 3-61.

Simons, R. (1990). The role of management control systems in creating competitive advantage: New perspectives. Accounting, Organizations and Society, 15(1/2), 127-143.

Simons, R. (2000). Performance measurement \& control systems for implementing strategy-Text and cases. Upper Saddle River, NJ: Prentice Hall.

Thompson, J. (1967). Organizations in action. New York: McGraw-Hill.

Von Hippel, E. (1998). The sources of innovation. New York: Oxford Press University.

Vosselman, E. y van der Meer-Kooistra, J. (2009). Accounting for control and trust building in interfirm transactional relationships. Accounting, Organizations and Society, 34, 267-283. 
Wang, P., Wee, C. y Koh, P. (1998). Control mechanisms, key personnel appointment, control and performance of sino-Singaporean joint ventures. International Business Review, 7(4), 351-375.

Waterhouse, J. y Tiessen, P. (1978). A contingency framework for management accounting systems research. Accounting, Organizations and Society, 3(1), 65-76.

Williamson, O. (1985). The economic institutions of capitalism. New York: Free Press. 